\title{
Tsafon
}

Revue d'études juives du Nord

$79 \mid 2020$

Les Juifs du Nord et du Pas-de-Calais dans la Shoah

\section{De Malines à Auschwitz}

Déportation des Juifs et des Tsiganes du Nord de la France

\section{Laurence Schram}

\section{(2) OpenEdition}

Journals

Édition électronique

URL : https://journals.openedition.org/tsafon/2838

DOI : $10.4000 /$ tsafon.2838

ISSN : 2609-6420

Éditeur

Association Jean-Marie Delmaire

Édition imprimée

Date de publication : 1 juillet 2020

Pagination : 75-96

ISSN : $1149-6630$

Référence électronique

Laurence Schram, « De Malines à Auschwitz », Tsafon [En ligne], 79 | 2020, mis en ligne le 01 juillet 2020, consulté le 24 juin 2021. URL : http://journals.openedition.org/tsafon/2838 ; DOI : https:// doi.org/10.4000/tsafon.2838 


\title{
De Malines à Auschwitz
}

\author{
Déportation des Juifs et \\ des Tsiganes du Nord de la France
}

Laurence Schram*

Le 10 mai 1940, l'offensive allemande sonne le glas de la « drôle de guerre » qui a débuté en septembre 1939, suite à l'invasion de la Pologne par les nazis. L'armée française avait opté pour une stratégie défensive, mais le Blitzkrieg [guerre-éclair] démontre l'échec flagrant de ce choix. Les troupes allemandes ont percé le front le 15 mai 1940, avant de remporter les batailles sur la Somme et sur l'Aisne en juin. Le 10 juin, le gouvernement français quitte Paris.

Avant même la signature par le maréchal Pétain de l'armistice à Rethondes, le 22 juin, les départements du Nord et du Pas-de-Calais sont rattachés au Commandement militaire pour la Belgique et le Nord de la France, dirigé par le général von Falkenhausen.

Cette situation lie étroitement le sort des Juifs et des Tsiganes des deux départements à celui des déportés raciaux de Belgique.

\section{Une planification européenne}

La création d'un camp de rassemblement à Malines s'inscrit dans une planification concertée de la déportation des Juifs de France, des Pays-Bas et de Belgique. À l'origine, ce camp est réservé uniquement

\footnotetext{
${ }^{*}$ Senior Researcher, Kazerne Dossin, Malines, Belgique.
} 
aux Juifs. Ce n'est qu'à la fin de l'année 1943 que les Tsiganes y sont également internés en vue de leur déportation à Auschwitz-Birkenau.

Contrairement à Drancy ou Westerbork, le camp de Malines s'inscrit exclusivement dans la perspective de la déportation génocidaire. C'est son unique fonction, celle pour laquelle un camp de rassemblement y a été installé par la Sipo-SD [Sicherheitsdienst-Sicherheitspolizei, Service de Sécurité et Police de Sécurité], tandis que Drancy et Westerbork sont établis avant l'offensive allemande du 10 mai 1940, par les autorités nationales.

Drancy est ouvert par le gouvernement français pendant la « drôle de guerre », en septembre 1939, comme lieu de détention pour les communistes. Un mois plus tard, la Cité de la Muette devient un camp d'internement réservé à des «étrangers dangereux pour la Défense nationale et la Sécurité publique ${ }^{1}$, parmi lesquels de nombreux réfugiés juifs du Reich. En juin 1940, le camp est réquisitionné par la Wehrmacht qui y installe le Frontstalag $111^{2}$. Des prisonniers de guerre, des internés civils britanniques, canadiens, yougoslaves, grecs... y sont enfermés ainsi qu'un millier de civils français rapatriés d'Allemagne. Cette population est peu à peu transférée dans d'autres lieux de détention, notamment des Stalags, camps ordinaires pour prisonniers de guerre en Allemagne ${ }^{3}$.

En août 1941, Drancy inaugure sa fonction de « camp pour Juifs ». Le 20, une rafle vise initialement les hommes juifs à Paris. En trois jours, plus de 4230 Juifs âgés de 18 à 50 ans, des Polonais, des Roumains, des Italiens... mais aussi de nombreux Français sont appréhendés suite à une initiative de Theodor Dannecker, le SS responsable de la «Question juive » en France ${ }^{4}$. Dans cette première période d'existence du camp, les internés, quasi exclusivement des hommes, font généralement partie d'une élite sociale ou intellectuelle. Ensuite, Drancy endosse véritablement son rôle dans la «Solution finale » un mois après la grande rafle du Vel' d'Hiv' à Paris des 16 et 17 juillet 1942, quand l'autorisation de déporter les enfants est donnée et que les enfants sont également enfermés à Drancy.

\footnotetext{
${ }^{1}$ Décret-loi du 18 novembre 1939.

${ }^{2}$ Annette Wieviorka et Michel Laffite, À l'intérieur du camp de Drancy, Paris, Éd. Perrin, 2012, p. 17.

${ }^{3}$ Ibid., p. 17.

${ }^{4}$ Michel Laffitte, Le camp de Drancy, Encyclopédie en ligne des violences de masse, [en ligne], publié le 23 novembre 2009, consulté le 9 mai 2011, URL: http://www.massviolence.org/Le-camp-de-Drancy, ISSN 1961-9898.
} 
Le camp est géré par les autorités françaises (préfecture, police et gendarmerie), situation qui perdure jusqu'en juillet 1943. Ensuite, le nouveau commandant du camp, Aloïs Brunner, exclut l'administration française du camp. Seule une garnison réduite de gendarmes français assure encore la garde extérieure.

Le gouvernement néerlandais a fait construire le centraal Vluchtelingenkamp Westerbork [Camp central pour réfugiés Westerbork] dès août 1939, dans le but d'absorber le flux des fugitifs du Reich, nombreux à arriver aux Pays-Bas. En théorie, les détenus y sont rassemblés en vue d'une émigration en Palestine. La construction du camp n'est pas encore achevée lorsque les 22 premiers réfugiés juifs allemands y sont internés le 9 octobre 1939. En janvier 1940, le camp compte 167 internés. La population du camp s'accroît rapidement dès février 1940 et, au mois d'avril, le camp abrite 749 personnes ${ }^{5}$.

Après février 1942, la construction du camp reprend avec plus d'intensité : 24 baraquements prévus pour 300 prisonniers et quelques autres petits bâtiments annexes sont ajoutés ${ }^{6}$. La capacité d'accueil du camp augmente notablement, permettant l'hébergement de bien plus de personnes que les 1100 réfugiés présents en juillet $1942^{7}$. Pour réaliser les travaux d'agrandissement du camp, les entrepreneurs utilisent la main-d'œuvre juive internée.

Le $1^{\text {er }}$ juillet 1942, le camp est mis à la disposition de l'occupant. À cette occasion, sa dénomination officielle devient Polizeiliches Judendurchgangslager Westerbork [Camp de transit policier Westerbork $]^{8}$. Le périmètre est alors délimité par des barbelés ${ }^{9}$. La gestion du camp dépend des autorités néerlandaises jusqu'en juillet 1942, du ministère de l'Intérieur d'abord et du ministère de la Justice ensuite. À partir de ce mois, la Sipo-SD, assistée par un SS-Wachbataillon (une formation de Waffen-SS néerlandais chargés entre autres de la surveillance des camps) ainsi que par la maréchaussée néerlandaise assurent la gestion et la garde de Westerbork. Lorsque la Sipo-SD en

\footnotetext{
${ }^{5}$ Harm Van Der Veen, Westerbork 1939-1945 - Het verhaal van vluchtelingenkamp en Durchgangslager Westerbork, Westerbork, Herinneringscentrum Kamp Westerbork, 2003, p. 11.

${ }^{6}$ Nanda van der Zee, Westerbork - Het Doorgangskamp en zijn commandant, Soesterberg, Uitgeverij Aspekt, 2006, p. 249.

${ }^{7}$ Harm Van Der Veen, Westerbork 1939-1945, op. cit., p. 21.

${ }^{8}$ Nanda Van der Zee Nanda, Westerbork, op. cit., p. 14.

${ }^{9}$ Harm Van Der Veen, Westerbork 1939-1945, op. cit., p. 53.
} 
prend le contrôle, le commandant néerlandais, Schol, reste encore en place jusqu'en janvier 1943.

\section{La genèse du camp de rassemblement}

Le 11 juin 1942, à Berlin, le Reichsicherheitshauptamt [Office Central de la Sécurité du Reich] planifie les déportations de la Zone Ouest, qui inclut la France, les Pays-Bas et la Belgique. Les responsables de la «Question juive » dans leur zone d'occupation respective, Theodor Dannecker, de Paris, Willy Zoepf, de La Haye, et Kurt Asche, de Bruxelles, participent à cette conférence avec le lieutenant-colonel SS Adolf Eichmann, chef du service IV B 4, la section juive de la Gestapo du Reich. Eichmann leur impose les quotas d'une première phase de déportation $^{10}$ : 40000 pour les Pays-Bas, 40000 pour la France et 10000 pour la Belgique dans un délai d'un mois. Cette première « évacuation » des Juifs se fait sous couvert d'une mise au travail. D'emblée, Kurt Asche, officier SS des affaires juives à Bruxelles, peut inclure un quota de $10 \%$ de Juifs inaptes au travail.

Quand les nazis décident d'installer un camp de rassemblement sur le territoire belge pour y interner les Juifs, la caserne Dossin de SaintGeorges à Malines est un choix évident. Cette ancienne caserne de l'armée belge se situe à mi-chemin entre Bruxelles et Anvers où réside la quasi-totalité des 70000 Juifs du pays. Elle est longée par la ligne de chemin de fer qui relie ces deux villes et se situe non loin d'un embranchement vers Louvain, l'Allemagne et l'Est. Très peu d'hommes suffisent à la surveiller efficacement. Le bâtiment encore partiellement meublé est suffisamment vaste pour y interner 2000 personnes. La proximité du camp de Breendonk, réservé aux prisonniers politiques, aux réfractaires et aux hommes juifs autorise l'occupant à considérer que l'environnement sécuritaire est relativement neutre, que la présence des internés ne provoquera pas un émoi particulier dans la population locale.

Le 15 juillet 1942, l'administration militaire allemande met la caserne Dossin à la disposition de la Sipo-SD pour y installer le camp de rassemblement ${ }^{11}$. Le même jour, Harry von Craushaar, commandant en

\footnotetext{
${ }^{10}$ Centre de Documentation juive contemporaine (CDJC), CXX-34 - NG-183, Lettreexpress d'Adolf Eichmann à Franz Rademacher, concernant la mise au travail des Juifs de France, de Belgique et des Pays-Bas, Berlin, 22 juin 1942.

${ }^{11}$ Archives générales du Royaume de Belgique, Auditorat militaire, Procès Schmitt, Attestation relative à la mise sur pied du camp de rassemblement de Malines, Harry von Craushaar, Bruxelles, 15 juillet 1942.
} 
second de l'Administration militaire, charge le SS-Sturmbannführer [major] Philipp Schmitt d'organiser le camp et d'en prendre la direction. Membre du parti nazi depuis 1925, Schmitt est déjà commandant à Breendonk. Là, ce dernier s'est déjà forgé une réputation de terreur et de cruauté.

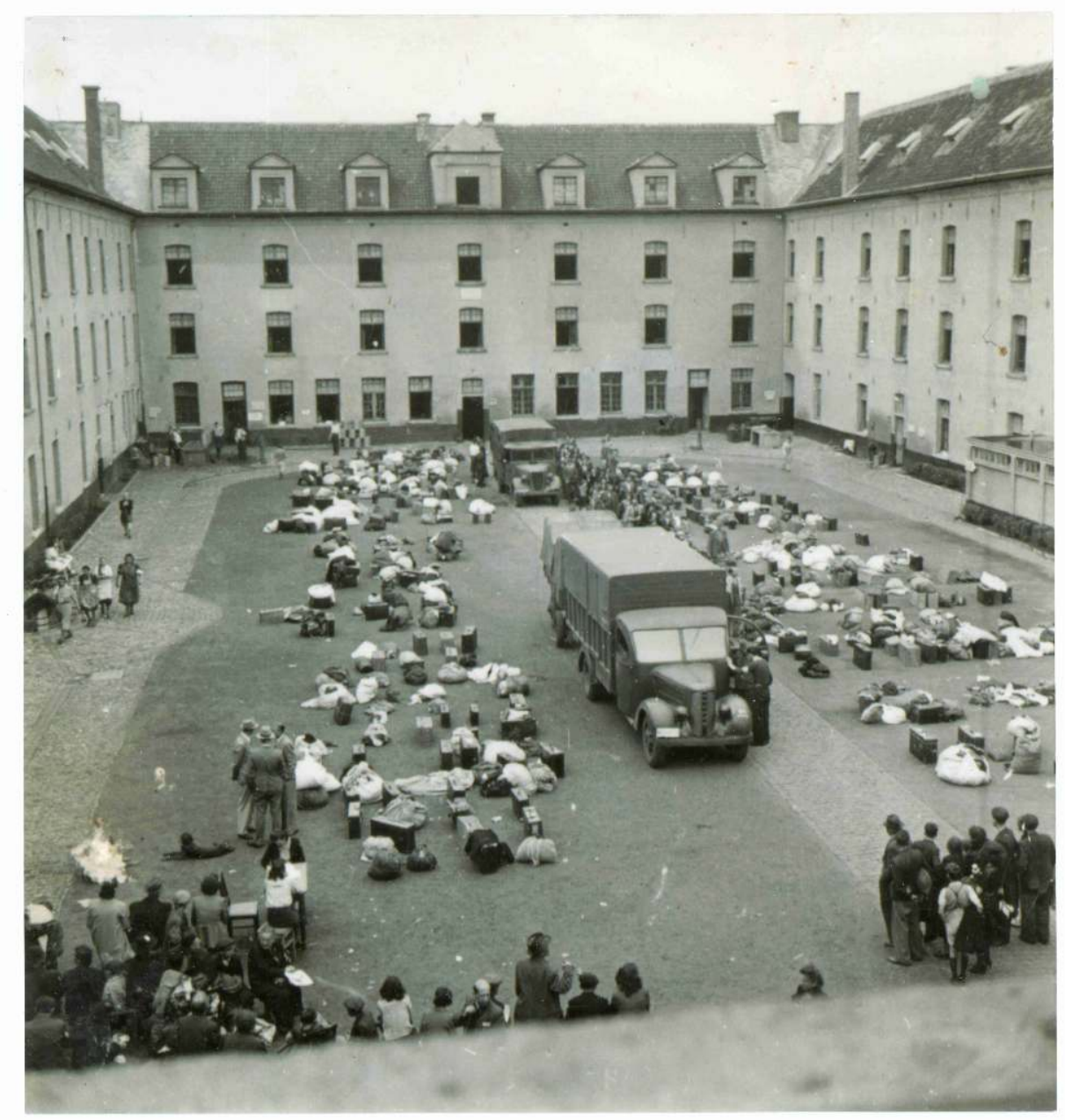

L'arrivée des convoqués dans la cour de la caserne Dossin, vers le 27 juillet 1942

Kazerne Dossin. Fonds Kummer

Lors d'une inspection effectuée en septembre 1941 par le chef de l'administration militaire et SS à titre honoraire, le général Eggert Reeder en personne, le constat est implacable. Reeder s'inquiète du traitement réservé aux détenus et des nombreux décès survenus à Breendonk. Suite à cette visite, Reeder réprimande sérieusement le major SS Constantin 
Canaris qui occupe alors le poste de chef de la police SS. Il lui déclare que le général von Falkenhausen «ne souhaite pas que le camp passe dans l'histoire comme l'enfer de Breendonk $\|^{12}$. Malgré cela, à Malines comme à Breendonk, Schmidt, secondé par des gardiens SS chevronnés et toujours accompagné de son fidèle berger allemand, use de ses armes favorites: l'arbitraire et la brutalité. La dizaine de SS allemands en charge de la caserne est renforcée par autant de SS flamands. À peine une soixantaine de nazis allemands et belges suffisait à la gestion du camp. Cette organisation du camp de Malines dépendant entièrement des services de l'occupant marque une autre différence avec les camps de Drancy et Westerbork, puisqu'à aucun moment les autorités belges ne sont impliquées dans son fonctionnement.

Le 22 juillet 1942, la Sipo-SD d'Anvers appréhende plus de 164 usagers juifs, hommes et femmes, revenant de Bruxelles à leur descente du train en gare d'Anvers ${ }^{13}$. Ils sont chargés sur des camions et sont tous emmenés à Breendonk ${ }^{14}$ car le camp de rassemblement de Malines n'est pas encore opérationnel.

Le 27 juillet, quelques heures avant son ouverture, certains sont transférés à la caserne Dossin. Là, les SS assignent quelques hommes à l'entretien et à l'intendance du camp. Ils affectent plusieurs jeunes femmes à la Aufnahme [l'accueil], le centre névralgique du camp où sont enregistrés tous les entrants. Ces dernières maîtrisent l'allemand et la dactylo, et, accessoirement, sont plutôt jeunes et jolies ${ }^{15}$. Elles en constituent l'«ossature administrative». Le minutieux travail d'inscription des nouveaux arrivants sur les listes de transport rédigées par ces secrétaires nous permet aujourd'hui de connaitre avec une grande précision les identités des déportés de Malines. Cependant, ces listes ne mentionnent ni les lieux d'arrestation ou de domicile, pas plus que les nationalités. Ce qui impose à l'historien une recherche fastidieuse sur

\footnotetext{
${ }^{12}$ CDJC, CDXCII-1. Procès-verbal du 22 septembre 1941, dicté par Victor, du bureau de chef de l'administration du commandant militaire en Belgique et en France du Nord, $a / s$. de l'entrevue du 17.9.1941 de Canaris avec Reeder, chef de cette administration, sur les conditions d'internement des détenus juifs et non juifs à Breendonk. (3 f. xérogr.).

${ }^{13}$ «La bête nazie impitoyable», dans België Vrij, Antwerpen, nº 8, août 1942 ; Lieven Saerens, Vreemdelingen in een Wereldstad, Een geschiedenis van Antwerpen en zijn joodse bevolking (1880-1944), Tielt, 1996, p. 590.

${ }^{14}$ Nationaal Memoriaal Fort van Breendonk, Document 3.4, Wachtboekje van de SS periode 1/04/1942 tot 12/08/1942.

${ }^{15}$ Maxime Steinberg, L'étoile et le fusil, 1942 - Les cent jours de la déportation, Tome 2, Bruxelles, Vie ouvrière, 1984, p. 197.
} 
plus de 25000 fiches pour combler cette lacune. Les chiffres qui suivent ne constituent, par conséquent, que des indications minimales.

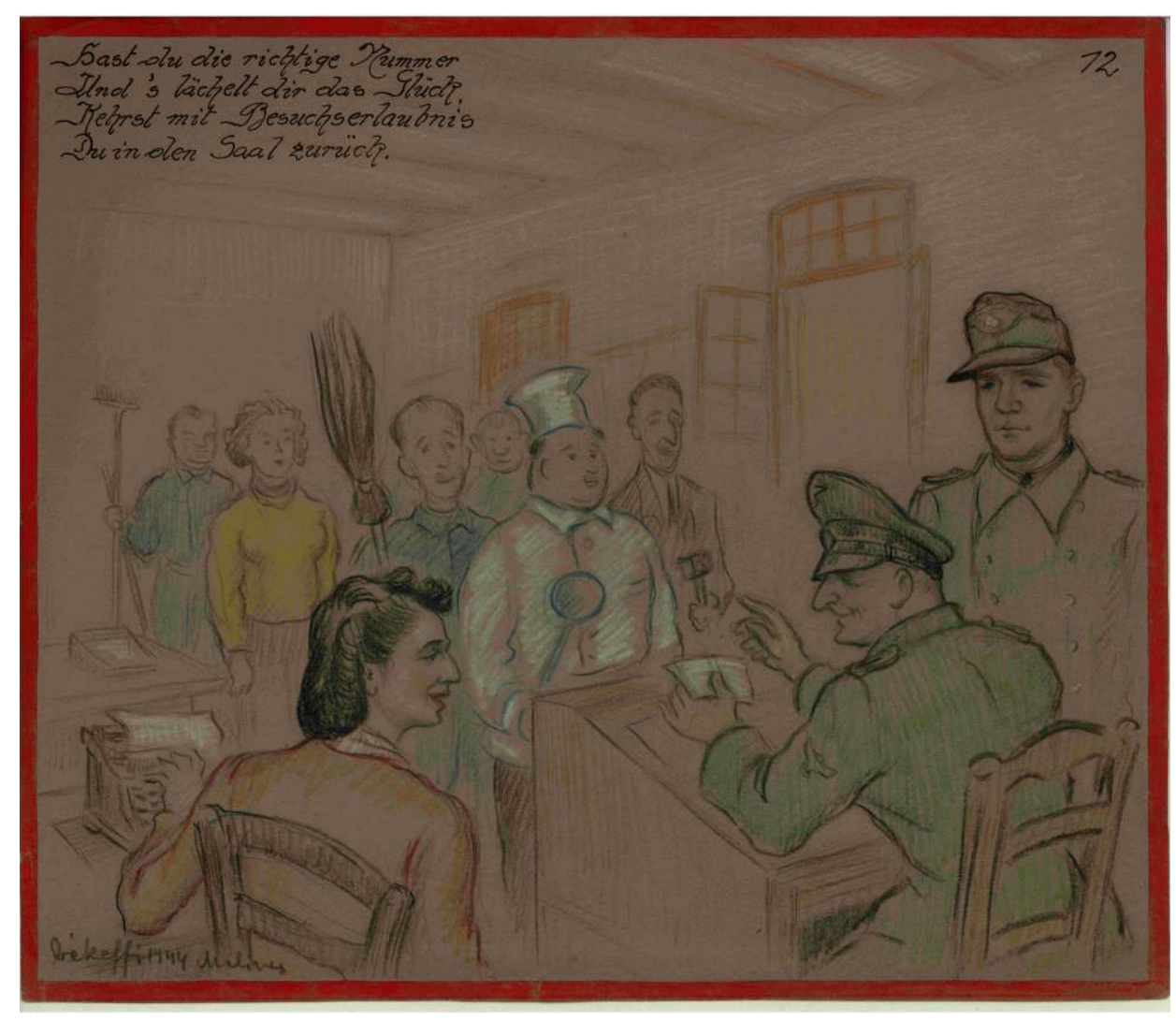

Scène de la Aufnahme

György Bekeffi, Malines, février 1944

NS-dokumentationszentrum der Stadt Köln/EL-DE Haus

\section{Le leurre de la convocation}

Deux jours avant l'ouverture du camp, l'occupant, dans une première phase, a fait distribuer plus de 12000 convocations par l'Association des Juifs en Belgique (AJB), l'équivalent belge de l'Union générale des Israélites de France et du Joodsche Raad [Conseil juif] aux Pays-Bas. La Sipo-SD ne dispose que d'une trentaine d'hommes pour procéder à l'évacuation des Juifs. Elle ne peut donc faire l'économie des relais « belges », les administrations belges, les forces policières locales, les sympathisants d'ordre nouveau, les militants de l'antisémitisme et l'AJB. 
Le recours aux convocations, sous le fallacieux prétexte d'une mise au travail obligatoire, s'avère être un échec. Plus de 12000 convocations sont distribuées principalement à des personnes en âge de travailler. La Sipo-SD compte, par ce biais, amener au camp de rassemblement de Malines les 10000 Juifs exigés par Berlin. Or, seulement 4000 personnes tombent dans le piège. Ce système est définitivement abandonné le 3 septembre 1942. Les derniers convoqués ont embarqué à bord du convoi VIII, le 8 septembre $1942^{16}$.

Même si le recours aux convocations n'a été appliqué qu'en Belgique, au moins 26 personnes du Nord de la France ont été déportées par le premier transport, le 4 août 1942. Ce petit groupe, qui inclut sept enfants de 4 à 12 ans, avait tenté de quitter Lille ou Lens pour se réfugier en zone libre. Arrêtés vers le 22/07/1942, les fugitifs sont emmenés à la prison de Loos, près de Lille, avant d'être transférés le 30 à Malines. Sur la liste du transport I, leurs noms sont inscrits sous les numéros 351 à 376, ce qui indique que tous y arrivent en même temps. Le plus jeune du groupe, le petit Paul Lobel, est accompagné de ses parents, Estera Reiss et Jacob Lobel, ainsi que par son grand frère Moïse, âgé de 8 ans. À leur suite figure une mère seule, Leopolda Rubinstein et ses trois enfants, Nelly, 11 ans, Fanny, 8 ans et Robert, 6 ans, vivant à Lens. De ces 26 personnes, seul Szaja Gruszkiewicz, 31 ans au moment de sa déportation, est rentré de déportation.

L'analyse de la liste du transport VII, dont le départ est prévu le $1^{\mathrm{er}}$ septembre 1942, révèle encore la présence de Juifs du Nord de la France. Ils sont inscrits au camp de rassemblement le 27 août sous les numéros 33 à 37, 39 et 49 . Ils sont au moins cinq de Lille et de Lens. Deux autres, Maximilien Cohen, $n^{\circ} 34$ et Rudolf Scherz, $n^{\circ} 39$, ont des domiciles parisiens, mais étant enregistrés dans la même série, on peut supposer qu'ils faisaient partie de ce groupe. Les deux survivants, Georges Priszkulnik et Joseph Macner ont été arrêtés à Lille, le 8 août 1942, comme probablement leurs compagnons d'infortune ${ }^{17}$.

\footnotetext{
${ }^{16}$ Laurence Schram, «Les convocations pour le travail à l'Est» dans Jean-Philippe Schreiber et Rudi Van Doorslaer (dir.), Les curateurs du ghetto. L'Association des Juifs en Belgique sous l'occupation nazie, Bruxelles, Labor, 2004, p. 319-344.

${ }^{17}$ Archives générales du Royaume de Belgique, Archives des Victimes de la Guerre (AGR-AVG), Dossier SDR 248321 de Joseph Macner, né le 15/10/1897 et Dossier SDR alphabétique de Georges Priszkulnik, né le 31/03/1924. Joseph Macner qui a le $\mathrm{n}^{\circ} 37$ dans ce convoi est l'oncle de Jeannette Rapoport, il habitait Cambrai (lire le témoignage de Jeannette Rapoport à la suite de ce dossier).
} 


\section{Le temps des rafles}

La seconde phase débute alors que l'envoi des convocations n'est pas encore abandonné. L'occupant constate son échec dès les premiers jours. Démentant ses prévisions, jamais 300 Juifs ne se sont présentés « volontairement» à la caserne Dossin ${ }^{18}$. Par conséquent, des méthodes plus radicales et plus violentes sont envisagées. Du 15 août 1942 au 11 septembre 1942, la Sipo organise de grandes rafles à Anvers (dans les nuits du 15 au 16 et du 28 au 29 août), une à Bruxelles (dans la nuit du 3 au 4 septembre) et une autre simultanément à Anvers et dans les régions de Lille, Lens, Douai et Valenciennes (du 11 au 12 septembre 1942). En tout, les grandes rafles de l'été 1942 amènent plus de 4330 victimes, dont des enfants, des femmes enceintes, des vieillards, à la caserne Dossin.

En France comme en Belgique, les nazis profitent de fêtes juives pour procéder à ces arrestations de masse. Ici, les familles juives étaient réunies pour célébrer Rosh Hashannah, le Nouvel An israélite. Arme au poing et sans ménagement, la Felgendarmerie et la police française arrachent les Juifs à leur domicile.

À Lens, les raflés sont rassemblés sur l'actuelle place Roger Salengro puis emmenés à la gare. Là, ils sont poussés dans des wagons de marchandises où se trouvent déjà les Juifs arrêtés à Douai. Aucune aide ne leur a été apportée par la population locale. Les victimes de Lens sont convoyées à la gare de Lille-Saint-Sauveur. Elles sont forcées de monter dans un train qui les conduit à Lille-Fives.

Par contre, à Lille, certains Juifs avaient été avertis du danger imminent et à la gare de Lille-Fives, des cheminots, des membres de la communauté protestante, des anonymes ont soustrait des personnes, dont des enfants, à leur sort ${ }^{19}$. Parmi eux, on trouve Maurice Baran, 9 ans, raflé chez lui, rue de la Vignette, le matin du 11 septembre, avec sa mère et son petit frère de trois mois, par les polices française et allemande. À la gare, Georgette Franchois, l'employée de maison de la famille, le retrouve et l'emmène dans un café à deux pas de la gare ${ }^{20}$, tandis que son petit frère est sauvé par une infirmière protestante. Le père de ces enfants, Abraham Baran, était l'un des déportés du transport I.

\footnotetext{
${ }^{18}$ AGR-AVG, United Restitution Organization (URO), «Aktenvermerk - Betreff : MAktion », Franz Mader, Anvers, 22 juillet 1942.

${ }^{19}$ Lire l'article de Monique Heddebaut dans ce dossier.

${ }^{20}$ Lire le témoignage de Maurice Baran-Marszak, à la suite de ce dossier.
} 
Le petit Josef Jozefowicz, né à Lens, âgé d'un mois et demi, est le plus jeune des déportés du transport X. Il est inscrit sur la liste de déportation avec ses parents, Léa Geldner et Idel Jozefowicz. Ce couple de tailleurs, comme la plupart des Juifs capturés pendant cette rafle, a immigré de Pologne. Le déporté le plus âgé est aussi issu de cette immigration de Pologne en France : Moïse Aschkenazy a 79 ans. Il est évident que ni le nourrisson ni le vieillard n'avaient aucune chance de survie à l'issue de la sélection. Tous deux ont été assassinés dans les chambres à gaz du centre de mise à mort de Birkenau, sans jamais passer par le complexe concentrationnaire d'Auschwitz-Birkenau.

Le bilan des arrestations dans le Nord de la France est lourd : les 11, 12 et 14 septembre 1942, au moins 513 Juifs du Nord de la France sont inscrits sur la liste de déportation du transport X. Aucune inscription n'est faite le dimanche 13 septembre, un jour de congé pour les SS de la caserne Dossin. Les raflés du Nord constituent presque la moitié de l'effectif de ce convoi de 1047 déportés.

Efficaces à première vue, les grandes rafles ont pourtant un effet que l'occupant n'avait pas prévu. La violence et la brutalité dont font montre la Sipo-SD et ses auxiliaires lors des arrestations poussent les Juifs dans la clandestinité. Ils sont désormais conscients du danger peutêtre mortel qui les menace.

Les deux systèmes précédents ayant montré leurs limites, les arrestations domiciliaires sur dénonciation et la chasse à l'homme menées par les membres de la Sipo-SD, leurs auxiliaires belges et même des limiers juifs, s'intensifient. La délation et la traque existaient évidemment dès le début des déportations. Désormais, elles restent pratiquement les seules façons de s'emparer des Juifs.

\section{Passage-éclair dans l'antichambre d'Auschwitz}

La fonction fondamentale de la caserne Dossin est celle de l'administration de la déportation raciale. À ce titre, le SS-Sammellager für Juden constitue bien l'antichambre de la mort pour $95 \%$ des déportés. La Aufnahme, établie au rez-de-chaussée de l'aile gauche du bâtiment, est le point névralgique du camp de rassemblement. Deux services y fonctionnent. Dans le premier, les dactylos juives enregistrent les nouveaux arrivants dès leur entrée au camp. Elles saisissent les papiers d'identité et distribuent des plaquettes en carton que tous les internés doivent porter autour du cou. Ces documents indiquent le 
transport et le numéro d'inscription sur les listes de déportation. Les secrétaires prennent les papiers d'identité, dressent les listes de transport et confisquent le contenu des portefeuilles et des poches, sous la direction du SS Max Boden ${ }^{21}$.

Dans le second, l'expert-comptable Erich Crüll, le délégué de la Brüsseler Treuhandgesellschaft [Société fiduciaire bruxelloise], met le point final à la spoliation des détenus. Dans une pièce séparée de la Aufnahme par un paravent, des internés et leurs effets sont fouillés très méticuleusement afin de s'emparer des objets de valeur ou des devises qu'ils n'auraient pas déjà remis. Tous ne subissent pas le contrôle corporel qui s'applique à ceux que l'on soupçonne de cacher des valeurs, les femmes juives, surtout les plus jeunes et les plus jolies, les Juifs religieux et les fortes têtes.

Dès les premiers instants au Sammellager, les internés sont brisés moralement et physiquement. Ils sont privés de toute dignité, désindividualisés et déshumanisés. L'arrivée se passe rarement sans violence, sans humiliations, sans coups. Parfois, les SS se livrent à des attentats à la pudeur.

Une fois achevées les formalités de la Aufnahme, les détenus sont conduits dans des chambrées du $1^{\text {er }}$ et du $2^{\mathrm{e}}$ étage, en fonction de leur numéro d'inscription sur les listes de transport. La plupart sont parqués dans des salles jonchées de paillasses jamais rafraîchies. Hommes, femmes et enfants s'y entassent de 100 à 130, sur des paillasses à même le $\operatorname{sol}^{22}$. Quelques privilégiés (travailleurs, Juifs de nationalité belge, cas douteux, mariages mixtes...) sont logés dans des salles moins rudimentaires et moins peuplées. Jan Hirsch, protégé par sa nationalité belge, les décrit :

\footnotetext{
Nous étions dans une salle où dormaient dans des lits de l'armée, entre 60 et 63 personnes. Les lits étaient très rapprochés. Ces salles étaient des greniers qui normalement auraient dû contenir une vingtaine de lits. De ce fait l'air était raréfié et vicié. Il y a toujours eu des poêles dans les salles, mais on ne recevait rien pour faire du feu. Ceci était d'application pour toutes les salles où il $\mathrm{y}$ avait des Belges et les mariages mixtes et le personnel. ${ }^{23}$
}

\footnotetext{
${ }^{21}$ Laurence Schram, Dossin, l'antichambre d'Auschwitz, Racine, Bruxelles, 2017, p. 107-119.

${ }^{22}$ AGR-AVG, Dossier prisonnier politique P 161 379/E 1787 au nom de Bacman Albert (Abram), 12/06/1906, Rezina, Note explicative d'Abram Bacman, 1948.

${ }^{23}$ AGR-Auditorat militaire (AM), Procès Boden, Farde 3, Section 1 - Affaires Vanderham Bernard et Israels Betty, doc. $\mathrm{n}^{\circ} 70$, Interrogatoire de Jean Hirsch, Bruxelles, 7 avril 1949.
} 


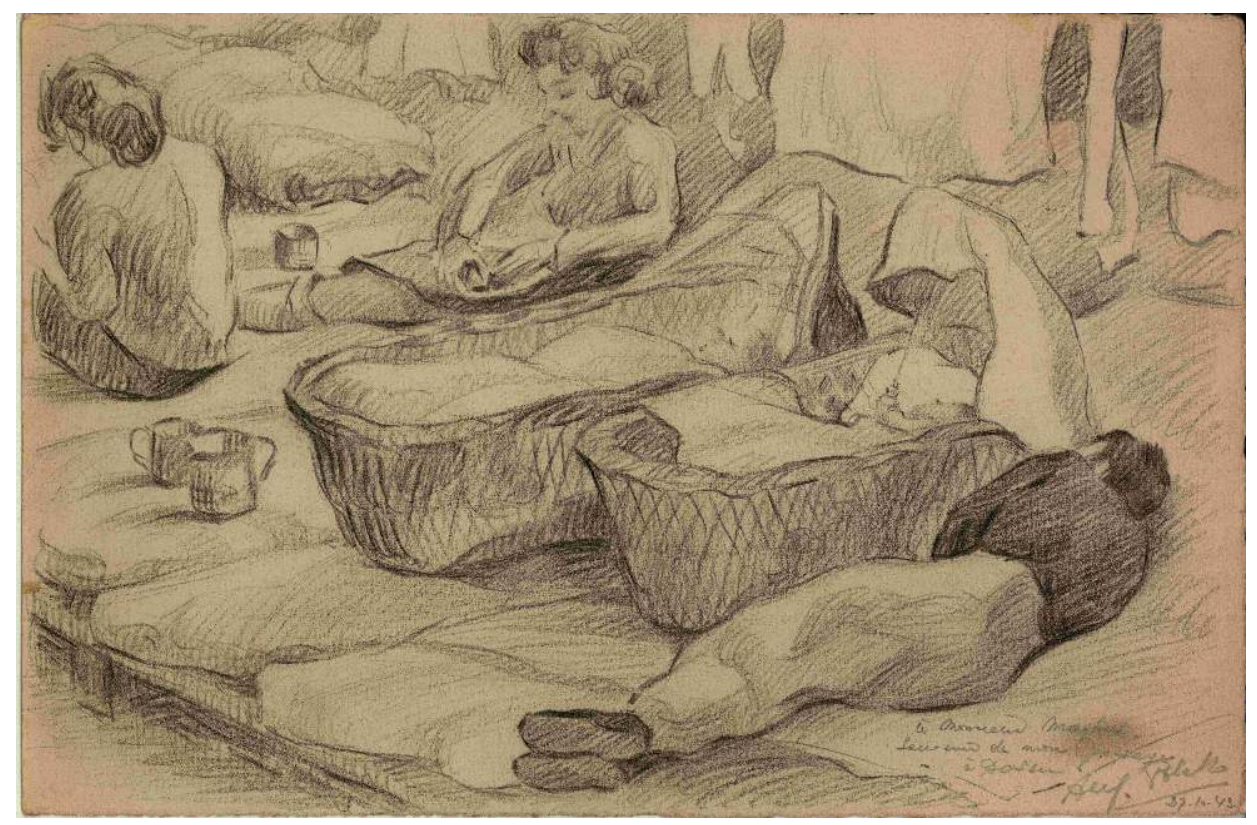

Une chambrée de Transportjuden

Alexandre Polak, Malines, 27 octobre 1943

NS-Dokumentationszentrum der Stadt Köln/EL-DE Haus

L'hygiène pose un autre souci aux Transportjuden [les Juifs enregistrés sur une liste de transport], qui vivent dans la plus grande promiscuité $^{24}$. Avant le départ du transport X, la population du camp compte probablement plus de 1200 prisonniers : 1047 qui doivent partir avec ce transport, une partie des futurs déportés du transport suivant, une centaine de Juifs relevant de statuts spéciaux ou membres du personnel juif du camp. Or, il n'y a que deux salles d'hygiène, l'une réservée aux hommes, l'autre aux femmes et aux enfants. Elles sont pourvues de longs éviers avec des robinets d'eau froide.

L'accès aux toilettes - une trentaine de cuvettes seulement - est limité aux deux promenades quotidiennes. En dehors de ces moments, il faut obtenir la permission d'un SS compatissant. Les internés s'y précipitent, créant toujours la cohue et les bousculades. La nuit, tous doivent se contenter des tinettes disposées sur le palier de l'étage. Malgré cela, seuls 57 décès surviennent à Dossin, un nombre remarquablement

\footnotetext{
${ }^{24}$ L. Schram, Dossin..., op.cit., p. 157-161.
} 
bas $^{25}$. Malgré ces conditions inhumaines d'internement, les coups et autres maltraitances, on ne meurt pas au camp de rassemblement. On n'en a pas le temps.

Les détenus sont soumis à un régime de famine, surtout dans la première période d'existence du camp ${ }^{26}$. Les SS et leurs aides prélèvent ce qui leur fait envie sur les maigres portions. Une à deux fois par semaine, l'AJB amène des colis qu'elle a préparés ou qui sont envoyés par les proches des détenus. Mais dans la période des convocations et des rafles, ces paquets arrivent souvent au camp après le départ du transport. Il est vraisemblable que les raflés du Nord de la France, qui n'ont passé que moins de quatre jours à Dossin, en aient été dépourvus au moment d'embarquer dans le train.

\section{Le transport $X$ à Auschwitz-Birkenau}

Le 15 septembre 1942, 1047 Juifs sont déportés du camp de rassemblement à Auschwitz en train de voyageurs de $3^{\mathrm{e}}$ classe. Premier convoi de plus de mille personnes, il achève, avec deux semaines de retard, le programme de déportation de 10000 Juifs fixé le 11 juin à Berlin.

Le 17 septembre, 405 hommes, 133 garçons, 413 femmes et 96 filles débarquent sur la alte Judenrampe [la vieille rampe des Juifs], un lieu en pleine campagne, au-delà de la gare d'Oświęcim, à mi-chemin entre le camp principal et Birkenau. Là, les SS effectuent immédiatement la sélection et condamnent 716 hommes, femmes et enfants jugés inaptes au travail ou simplement surnuméraires, à l'assassinat immédiat dans les bunkers du centre de mise à mort de Birkenau. Ces deux fermettes, sises à l'arrière du camp de concentration de Birkenau et en dehors de son périmètre, ont été transformées en chambres à gaz. L'effectif du transport incluait 229 enfants de moins de 15 ans et 194 personnes ayant dépassé 50 ans. Plus de $80 \%$ des femmes et des filles du transport $\mathrm{X}$ sont asphyxiées dans les bunkers, tandis que la proportion d'hommes voués au même sort est bien inférieure : $57 \%$.

Le taux d'extermination immédiate de ce convoi dépasse $68 \%$.

Les SS de la solution finale retiennent 331 esclaves pour l'extermination par le travail : 230 hommes sont identifiés dans la série allant du 64005 au 64234 et 101 femmes sont immatriculées par les

\footnotetext{
${ }^{25}$ Ibid., p. 169.

${ }^{26}$ Ibid., p. 149-153.
} 
numéros 19821 à 19 921. Une fois tatoués, ces 331 Häftlinge [déportés versés dans l'univers concentrationnaire d'Auschwitz-Birkenau] sont confrontés à l'épuisement par le travail, à la faim, aux mauvais traitements, aux épidémies. La mort fait des ravages parmi eux.

Dans les séries lacunaires des Sterbebücher ${ }^{27}$ [recueil des actes de décès], on retrouve 81 actes de décès pour le transport $\mathrm{X}$ : onze concernent des femmes et 70 , des hommes. Le premier document est établi le 22 septembre 1942 au nom de Rosa Reichmann, née à Leipzig en 1909. Raflée à Anvers au cours de la troisième action de masse, elle ne résiste pas plus d'une semaine au régime qui lui est infligé à Birkenau. Le décès le plus tardif, dont on a la trace, est celui de David Deutsch, un boulanger anversois de 24 ans, pris dans la même rafle du 11 septembre. Il meurt le 13 mars 1943, après moins de six mois passés au camp.

Il est remarquable que 43 décès surviennent dans le courant du seul mois d'octobre 1942. Ils sont vraisemblablement causés par l'importante épidémie de typhus qui s'est propagée à Auschwitz et ses annexes dès le 30 septembre. Un autre pic de mortalité est atteint en janvier 1943. Tous les actes retrouvés pour cette période concernent uniquement des hommes. Comme l'auteur le fait remarquer, « ce mois-là, de lourds travaux d'agrandissement du camp conjugués à des sélections quasi quotidiennes dans les Blocks et à une nouvelle épidémie de typhus, touchant principalement Buna-Monowitz, augmentent la mortalité interne au camp $»^{28}$. Buna-Monowitz est l'un des plus importants camps annexes d'Auschwitz. L'analyse des dossiers des survivants de la déportation de Malines à Auschwitz révèle que de nombreux hommes déportés de Belgique et aptes au travail y ont été utilisés comme forçats.

Des 331 Juifs retenus pour le travail, on recense 26 survivants, 23 hommes et 3 femmes. Quinze d'entre eux, dont une seule femme, Frieda Thau, avaient été raflés dans le Nord de la France.

\section{Quelques données sur les autres transports}

L'analyse des listes suivantes permet encore d'identifier au moins 10 déportés de Lille et Lens, enregistrés le 25 septembre 1942 pour le transport XI, sous les numéros 2291 à 2299. Les familles Sosnowicz et

\footnotetext{
${ }^{27}$ Państwowe Muzeum Auschwitz-Birkenau, Sterbebücher.

${ }^{28}$ Maxime Steinberg et Laurence Schram, «Malines-Auschwitz: 1'historique des transports 1942-1944 » dans Mecheln-Auschwitz-1942-1944, vol. 1, Bruxelles, VUBPress, 2009, p. 291.
} 
Rosenblum ont peut-être été arrêtées lors de la rafle du 11 septembre, mais livrées plus tard au camp de rassemblement.

Le 19 avril 1943, au moins quatre détenus originaires du Nord de la France embarquent à bord d'un transport composé pour la première fois de wagons de marchandises. Herman Goldschmidt, enregistré au numéro 842, Maurice Swiercz et Rachel-Ruchla Galeck, aux numéros 1382 et 1383 ont un domicile à Lille. Le ${ }^{\circ} 1209$ est attribué à Alfred Beiline, un Lensois.

Herman Goldschmidt et Alfred Beiline, âgés de 19 ans, s'évadent du convoi XX. Peut-être ont-ils profité de l'attaque de la résistance à moins qu'ils n'aient profité des outils dissimulés dans la paille de certains wagons par des travailleurs juifs de la caserne Dossin. Si le premier échappe définitivement aux griffes des SS, il n'en va pas de même pour Alfred Beiline. Arrêté de nouveau et ramené au SSSammellager, il est inscrit avec d'autres évadés sur une liste annexe du transport XXI et déporté le 31 juillet 1943, cette fois sans retour.

\section{Octobre 1943 : une traque particulière}

En octobre 1943, plusieurs arrestations domiciliaires sont encore menées en Belgique comme dans le Nord de la France. Elles ciblent des Juifs possédant certaines nationalités particulières. Cent d'entre eux sont arrêtés durant ce mois, dont au moins 15 à Croix, Roubaix, Lille et Quiévrechain.

Les nationaux hongrois, turcs ou espagnols relevaient jusqu'ici d'un régime particulier, lié aux relations diplomatiques et militaires entre leurs pays d'origine et le Reich nazi. Quand la solution finale est mise en œuvre en Belgique et dans le Nord de la France, ils bénéficient de mesures d'exception. Ces exemptions s'achèvent en même temps que leur alliance avec le Reich ou la neutralité de leur pays. Quoi qu'il en soit, cette déportation ne s'inscrit pas dans le schéma de la déportation génocidaire de Malines à Auschwitz-Birkenau. Pour résumer, on pourrait dire que ces personnes sont « déportables », mais pas « exterminables ${ }^{29}$.

Les Juifs hongrois, turcs ou espagnols sont dirigés en transports exceptionnels, désignés par une lettre « $Z »$, vers des camps de

\footnotetext{
${ }^{29}$ Laurence Schram, La caserne Dossin à Malines 1942-1944 - Histoire d'un lieu, Thèse présentée en vue de l'obtention du grade académique de Docteur en Histoire, Histoire de l'Art et Archéologie sous la direction du Professeur Pieter Lagrou, Université libre de Bruxelles, Bruxelles, 2014-2015, p. 484.
} 
concentration. La signification $\mathrm{du}\langle\mathrm{Z} »$ demeure inconnue. Il ne se réfère ni au « $\mathrm{Z} »$ des Tsiganes (Zigeuners) ni à « Sondertransport» (transport spécial) qui s'écrit en allemand avec un « $\mathrm{S} »$.

À la caserne Dossin, ces derniers sont en quelque sorte des privilégiés. Logés dans des salles une peu moins surpeuplées et un peu mieux équipées que les Transportjuden. Des longues tables, des bancs, des petites étagères, des lits en fer s'y trouvent toujours, reliquats du temps où l'armée belge était maître des lieux. Comme tous les détenus, ils ont le droit de recevoir des colis de l'extérieur et bénéficient du ravitaillement fourni par le Secours d'Hiver, une organisation caricative.

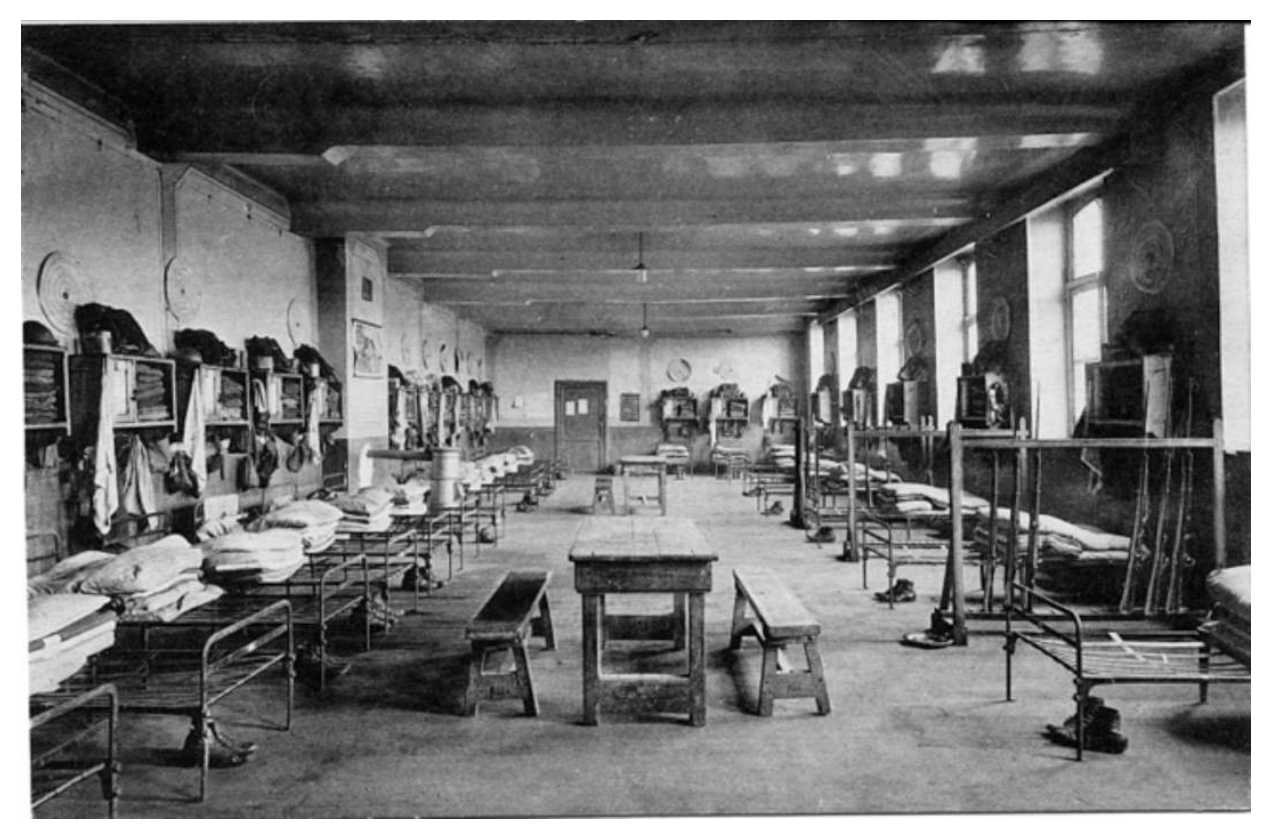

Une chambrée de la caserne Dossin, 1932 Collection privée Jan Somers

La Judenabteilung de Bruxelles organise ce premier transport spécial formé d'un très petit nombre de personnes ${ }^{30}$. Cette fois, les déportés n'embarquent pas à Malines, mais sont conduits jusqu'à Bruxelles, à la gare du Nord, dans des wagons de voyageurs.

Le 13 décembre 1943, 132 Juifs hongrois, espagnols et turcs sont déportés en Allemagne, les 64 femmes et les enfants à Ravensbrück et les 68 hommes à Buchenwald. La famille de Lili Rosenberg, mieux connue

\footnotetext{
${ }^{30}$ AGR-AM, Procès Boden, Farde 1, Section IV-B - P.V. Audition de sujets allemands, doc. $n^{\circ} 27$, Procès-verbal d'interrogatoire de Frank Hans, 31 mars 1949, Bruxelles.
} 
aujourd'hui sous le patronyme de Lili Leignel, est capturée le 30 octobre comme le reste de ce groupe. Le 15 décembre 1943, ils arrivent à leurs destinations respectives. N'étant pas intégrés dans la « solution finale», Ravensbrück et Buchenwald ne sont pas dotés de centres de mise à mort pas plus que d'installation de gazage. Tous les déportés y sont internés et identifiés comme «DIKAL» (Darf in kein anderes Lager). Dans le jargon de la Sipo-SD, cela signifie qu'ils ne peuvent être déplacés de leur camp de destination. Les nazis avaient l'intention de se servir d'eux comme monnaie d'échange: des Juifs contre des prisonniers de guerre allemands.

À Buchenwald et ses dépendances, les hommes sont identifiés comme des «Politische Türke Juden», des prisonniers politiques turcs et juifs. Ils y sont affectés à des travaux lourds: installation de câbles, construction de bunkers et d'égouts, déchargement de marchandises... Les conditions de vie sont catastrophiques et le typhus sévit. La santé des déportés s'y dégrade rapidement. Ceux qui sont considérés comme inaptes au travail sont transférés à Bergen-Belsen ou directement à Auschwitz.

À Ravensbrück, la situation est légèrement moins meurtrière. Quelques femmes et enfants turcs ont été échangés contre des prisonniers de guerre allemands en Turquie ${ }^{31}$.

Le taux de survie de $56 \%$ est sans commune mesure avec celui de $5 \%$ des convois génocidaires d'Auschwitz-Birkenau. Il souligne toute la différence qui existe entre un camp de concentration et un centre de mise à mort. Chose exceptionnelle, 14 des 15 enfants de ce transport ont survécu $^{32}$ alors que seuls 14 des 4245 enfants déportés à AuschwitzBirkenau ont été rapatriés.

\section{Les Tsiganes à la caserne Dossin}

Lors de sa création, le SS-Sammellager für Juden était, comme son nom l'indique, un camp de rassemblement réservé aux Juifs. À la fin du mois d'octobre 1943, les Sipo-SD de Bruxelles et de Lille raflent les populations tsiganes dans leurs territoires respectifs. Puisque la destination de leur déportation est également Auschwitz-Birkenau, ils sont envoyés à la caserne Dossin ${ }^{33}$.

\footnotetext{
${ }^{31}$ Lire le témoignage de Zelda-Simone Segouriano, à la suite de ce dossier.

${ }^{32}$ Dont Lili Leignel (née Rosenberg) et ses deux frères, ainsi que leur maman.

${ }^{33}$ Lire l'article de Monique Heddebaut, dans ce dossier.
} 
Les familles arrivent à Malines en plusieurs groupes du 5 novembre 1943 au 12 janvier 1944. Le 6 décembre, 166 Tsiganes sont déjà inscrits sur la liste du transport Z. Le 9 décembre, 182 Tsiganes sont amenés à la caserne Dossin. Le 12 janvier 1944, la liste du transport Z est clôturée.

Le 6 décembre, le camp de Malines a déjà enregistré 166 nouvelles entrées. Le 9 décembre, 182 hommes, femmes et enfants sont ajoutés à la liste du transport Z. D'autres ont transité par les prisons de Loos ou de Lille avant d'être livrés à Malines.

Une fois internés, les Tsiganes sont enregistrés sur la liste par des employés de la Kripo, la police criminelle, et deux dactylos de la section $\mathrm{V}$ de la Sipo-SD, en charge de la répression des crimes $^{34}$. Venus spécialement, ces derniers travaillent pendant deux semaines à la confection de cette liste. Cette procédure est exceptionnelle. Habituellement, ce sont les secrétaires juives du camp qui établissent ces documents, mais la répression des Tsiganes relève de la responsabilité de la Kripo.

L'arrivée des Tsiganes ne passe pas inaperçue. La présence de si nombreux enfants, presque la moitié du groupe, choque les internés juifs. Irène Spicker, depuis la fenêtre de la salle des peintres, décrit la scène :

Les coups de sifflet retentissaient de tous côtés. 'Tout le monde en haut!' Une demi-douzaine de camions roulait dans la cour. Des gens avec des possessions pitoyables et une foule d'enfants ont été conduits dans l'une des cages d'escaliers, sans le passage habituel par la Aufnahme. ${ }^{35}$

Eva Fastag, la secrétaire juive de la Aufnahme, le bureau d'enregistrement du camp, voit «les colonnes franchir le portail de la caserne. C'était pareil aux autres. Mais ils étaient encore plus misérables. Ils faisaient encore plus pitié. Surtout qu'ils avaient une ribambelle d'enfants $»^{36}$.

\footnotetext{
${ }^{34}$ AGR-AM, Procès Boden, Farde 2, Section 8 - Témoins généraux $(H)$, doc. no 9 , Déposition de Heiber Mojzesz, Bruxelles, 25 février 1949.

${ }^{35}$ Irène Awret, They'll have to catch me first - An artist's coming of age in the third Reich, London, University of Wisconsin Press, 2003, p. 280. [« Whistles were blowing from all sides. 'Everybody upstairs!' Half a dozen trucks rolled into the yard. People with pitiful belongings and a crowd of children were driven into one of the stairwells without the customary registration at the Reception ».]

${ }^{36}$ Entretien téléphonique du 26 décembre 2005 entre Monique Heddebaut et Eva Fastag-Dobruszkes et validation du témoignage par les correspondances des 18 janvier et 26 février 2006.
} 
Avant d'être menés dans les chambrées, les SS confisquent leurs biens, leurs bijoux et leurs instruments de musique.

Les conditions de détention que les Tsiganes endurent à la caserne Dossin sont effroyables ${ }^{37}$. Ils sont confinés à 352 dans une ou deux salles du premier étage, au-dessus des cuisines du camp. Les témoignages divergent à ce sujet ${ }^{38}$. La porte de leur salle est fermée à clé, clé détenue par un garde SS en faction devant leurs dortoirs. Privés de colis extérieurs, tous sont affamés. Hommes, femmes et enfants dorment entassés sur des paillasses crasseuses dans une chambrée surpeuplée.

Privés d'accès aux toilettes, les Tsiganes doivent se contenter de trop peu de tinettes placées dans leurs salles. Ces seaux hygiéniques, qui débordent et empestent l'air, sont trop peu vidés et nettoyés. L'accès aux douches est prohibé sauf quand les SS en décident autrement. Alors, ils contraignent les Tsiganes à se rendre dans les salles d'eau où hygiène se confond avec brimades et humiliations :

Parfois, on décidait que ce peuple aux pieds sales, couvert de vermine, avait besoin d'être lavé et étrillé. On les faisait entrer dans le lavoir, on les faisait se déshabiller et on jetait des seaux d'eau chaude sur leur peau sombre. Après quoi, ils pouvaient remettre leurs loques pouilleuses et retourner, pieds nus, patauger dans la boue. Cette promenade hygiénique ne durait pas tout à fait une heure. L'après-midi, ils avaient encore droit à une sortie du même genre. Après, c'était fini. Ils retournaient jusqu'au lendemain dans leur cage empuantie. ${ }^{39}$

Les Tsiganes ne peuvent sortir des dortoirs qu'une heure par jour, sans horaire régulier et toujours quand les Juifs sont déjà dans leurs chambrées.

Hélène Beer, Juive de nationalité belge et chroniqueuse de la vie du camp, fait une description éloquente de cette promenade toujours pénible, violente, humiliante :

À neuf heures et demie, après la promenade des Juifs, ils [les Tsiganes] avaient aussi droit à tourner en rond dans la cour. Un SS les tenait en joue avec sa mitraillette. Leur chef - prompt à les battre - marchait à leur côté, le fouet au poing. Même parmi les Tsiganes, la Gestapo avait trouvé un collaborateur. En

\footnotetext{
${ }^{37}$ L. Schram, Dossin ..., op.cit., p. 253-258.

${ }^{38}$ Dans une salle, selon les sources suivantes : A.M., Procès Boden, Farde 1, Section VI - Jugement et appel, P.V. de l'audience publique, doc. $\mathrm{n}^{\circ}$ 52, Déposition de Voss Alfred, Bruxelles, 3 juillet 1950 \& Kazerne Dossin, Audio, Interview de Mathilde Lipschutz, réalisée par Frédéric Molle, Anvers le 9 juin 1997. Dans deux salles, selon I. Awret, They'll have..., London, 2003, p. 280.

${ }^{39}$ Hélène Beer, Salle 1, Bruxelles, Charles Dessart, 1946, p. 43.
} 
tête marchaient trois violonistes. On leur remettait les instruments au début de la promenade, on les leur enlevait à la fin. Ils jouaient des airs d'opérettes et des valses qui résonnaient tristement dans l'air froid et sous la pluie. Un gamin de dix ans chantait. Il avait une voix ravissante et des haillons sordides. Derrière les musiciens venaient les femmes dont les larges jupes traînaient dans la boue. Elles portaient souvent des enfants sur le dos. Puis les hommes, toujours à l'affût. Leurs yeux noirs et mobiles tournaient à gauche tournaient à droite. ${ }^{40}$

Tandis que les musiciens sont contraints de jouer, les autres doivent marcher en cadence. Les SS leur distribuent des coups. Ces sorties sont pénibles, violentes et humiliantes. Aussitôt la promenade finie, tous sont ramenés et enfermés dans leur salle.

Et cette routine se répète pendant plusieurs semaines, jusqu'au départ de leur transport, le 15 janvier 1944.

Hélène Beer, qui a sans doute été chargée avec d'autres détenues juives de nettoyer les dortoirs, dresse un état des lieux éloquent :

C'était donc là qu'avaient vécu les Tsiganes, pendant un mois, dans cette salle sombre, aux fenêtres closes. Dès l'entrée, on tombait sur les tinettes. Elles encombraient un angle de la salle et débordaient. Nuit et jour, elles restaient dans la chambre et remplissaient celle-ci de leur puanteur. Cependant, comme si cela ne suffisait pas les dalles étaient couvertes d'excréments. Les paillasses étaient toutes éventrées, les gamelles et les pots noirs de fumée. ${ }^{41}$

Les Tsiganes quittent la caserne Dossin le 15 janvier 1944, conjointement avec le $23^{\mathrm{e}}$ transport de Juifs. Le voyage dure trois jours, dans des wagons à bestiaux, sans tinettes, avec trop peu d'eau et de pain.

\section{Un bilan}

Comme les camps de Drancy et de Westerbork, la caserne Dossin a joué un rôle fondamental dans la déportation des Juifs de Belgique : du 27 juillet 1942 au 4 septembre 1944, plus de 26000 Juifs y ont été internés pendant l'occupation allemande. La plupart des détenus (25 272 Juifs et 353 Tsiganes) ${ }^{42}$ ont été envoyés de ce camp de rassemblement vers Auschwitz-Birkenau. Seuls 1252 ont survécu à cette déportation, à peine $5 \%$ des déportés. Ce chiffre, révélateur de l'ampleur du judéocide, ne constitue qu'un léger bruit statistique. D'autres déportations depuis

\footnotetext{
${ }^{40}$ Ibid., p. 42-43.

${ }^{41}$ Ibid., p. 44.

${ }^{42}$ Tous les chiffres cités proviennent de la base de données Déportés de la Caserne Dossin, réalisée par Laurence Schram, état du 23 mars 2020.
} 
Malines, marginales et exceptionnelles, acheminent encore 218 Juifs vers les camps de concentration de Buchenwald, Ravensbrück ou BergenBelsen, ou encore au camp d'internement de Vittel. Après le 8 mai 1945, on identifie 146 survivants parmi eux, soit $67 \%$ de l'effectif de ces transports spéciaux. Le taux de survie de ces déportés-là est incroyablement élevé par rapport aux déportés de la « solution finale ».

L'été 1942 est bien la période cruciale où l'extermination des Juifs est perpétrée en Belgique comme dans le Nord-Pas-de-Calais. Plus des deux tiers des Juifs voués à la solution finale sont éliminés en 100 jours. Les survivants des transports I à XVII ne constituent que $2,5 \%$ de l'ensemble des rapatriés, soit un chiffre de moitié inférieur à la moyenne ( $5 \%$ pour l'ensemble de la déportation).

Au bilan final, $44 \%$ de la population juive de Belgique a été déportée entre 1942 et 1944. Les Juifs du Nord de la France perdent également la moitié de leur population.

Même si les Tsiganes ne sont pas visés par un génocide, leur sort n'en demeure pas moins meurtrier. En un seul transport, leur population en Belgique et au Nord de la France est réduite de $70 \%$. La répression qui les vise a été motivée par une politique raciste de sédentarisation et d'exclusion, menée tant par les nazis que par les autorités policières locales.

La particularité des déportations raciales du Nord de la France sous l'occupation nazie est encore trop souvent oubliée des historiens français et par conséquent moins étudiée.

La difficulté provient sans doute du fait qu'il s'agit là d'une histoire transfrontalière et que les chercheurs ont tendance à se limiter aux archives accessibles dans leurs pays respectifs. La mise en commun de nos savoirs et de notre expérience est indispensable pour pouvoir réaliser un tableau aussi riche et détaillé que possible. Une approche transnationale se justifie pleinement d'autant plus que les populations juives ou tsiganes sont très variées, notamment sur le plan des nationalités.

À partir d'une étude microhistorique de la caserne Dossin dans la période où elle fonctionne comme camp de rassemblement pour les déportés raciaux, le chercheur peut affiner sa vision des divers cas de figure, retracer les parcours variés des déportés, les vagues d'arrestations, les destinations de la déportation, le traitement réservé aux uns et aux autres une fois détenus à Malines, selon la classification dont ils relèvent: Transportjuden, Juifs de nationalités spécifiques, Tsiganes de 
France, de Belgique ou d'ailleurs. Car, comme le disait feu mon professeur Maxime Steinberg, historien pionnier de l'histoire de la Shoah en Belgique, il faut rendre à César ce qui est à César, tout ce qui est à César, ni plus, ni moins. 